\title{
Synthesis and Characterization of a Novel Class of Lewis Acid Doped Tetraaniline
}

\author{
K. BASAVAIAH* , and A.V. PRASADA RAO \\ Department of Inorganic and Analytical Chemistry \\ Andhra University, Visakhapatnam -530003, India \\ klbasu@gmail.com
}

Received 7 July 2011; Accepted 20 September 2011

\begin{abstract}
Boron trifluoride $\left(\mathrm{BF}_{3}\right)$ doped tetraaniline with novel structure were successfully synthesized via two step method. In the first step, emeraldine base form tetraaniline was synthesized via oxidative chemical polymerization of N-phenyl-1, 4-phenylenediamine using ammonium persulphate as oxidant. In the second step involves control $\mathrm{BF}_{3}$ doping of tetraaniline using boron trifluoride etherate. As synthesized $\mathrm{BF}_{3}$ doped tetraaniline have been well characterized by UV-Visible spectroscopy, Fourier transform infrared spectroscopy (FTIR), X-ray diffraction patterns (XRD), and scanning electron microscopy (SEM) and thermogravimetry. Thermogravimetry studies revealed that the $\mathrm{BF}_{3}$ doping improved the thermal stability of tetraaniline.
\end{abstract}

Keywords: Conducting polymers; Lewis acid; polymer supported catalyst; tetraaniline.

\section{Introduction}

With the discovery in 1960 of intrinsically conducting polymers (ICPs), an attractive subject of research was initiated because of the interesting properties and numerous technological application possibilities of ICPs. It was expected that ICPs would find their potential applications in multidisciplinary areas such as electrical, electronics, thermoelectric, electrochemical, electromagnetic, electromechanical, electro-luminescence, electrorheological, membrane, and corrosion protections, chemical and molecular sensors. ${ }^{1-9}$ However, many of the potential uses for ICPs have yet to be explored because of a number of obstacles that need to be overcome. Among the ICPs, polyaniline (PANI) is found to be the most promising because of its ease of synthesis, low cost monomer, and unique redox property in doped form, good processbility, highly conductive, and better chemical and environmental stability ${ }^{10-11}$ compared to other ICPs. Among various oxidized forms of PANI, $50 \%$ - oxidized PANI, i.e. emeraldine base Polyaniline (EBPANI) is the only redox form that become conducting in the doped state with different protonic acids. However, in this case the doping is an acid-base reaction in the Brönsted sense and consists of the protonation of the base form of emeraldine with a sufficiently strong protonic acid i.e. Brönsted acid. Due to the presence of the lone electron pair on the imine and amine sites, emeraldine is not only a Brönsted base, but also a Lewis base. Recently, K. Bieńkowski and his co-workers have demonstrated that polyaniline can be doped not only with Brönsted 
acids, but also with Lewis acids i.e. molecules with electron deficit capable of formation of a coordination bond with emeraldine via its lone pair of electrons. There have been several Lewis acids such as $\mathrm{AlCl}_{3}, \mathrm{SnCl}_{4}$, and $\mathrm{FeCl}_{3}{ }^{12-13}$ used as dopant for polyaniline. Among these Lewis acid dopants, $\mathrm{FeCl}_{3}$ and $\mathrm{SnCl}_{4}$ act as complexing agents of Lewis acid nature for polyaniline. Since they are too week oxidizing agents to be involved in a redox reaction with EBPANI. Lewis acid type doping is extremely important since the complexation of polyaniline with Lewis acids significantly alters the band structure of the polymer and leads to important changes in its electrical and optical properties. Lewis acid-doped PANI systems are expected to be different from the conventional protonated PANI owing to a qualitatively different chemical inter-action between the dopant and the polymer; for instance, the absence of any counter ion in these systems may have different influence on the properties.

Only a few scattered reports have appeared during the past century describing the synthesis of oligomers of aniline. It is surprising that the very little attention has paid to these extremely important species on which chemical and physical properties of polyaniline are based. Studies of the topological and geometrical isomers formed after oxidation of anile allow one to understand the complexity of the molecular interactions in polyaniline. The most interesting form of polyaniline is emeraldine, which contains ca. 50\% amine and ca. 50\% imine nitrogen, that is one of 4 rings is quinoid, the remaining three being benzoid. Hence, tetra PANI is perfect model compound for PANI since its chemical structure resemble to the EBPANI.

In this paper, we present the synthesis of boron trifluoride $\left(\mathrm{BF}_{3}\right)$ doped Tetraaniline. Firstly, EB form of Tetraaniline was synthesized via chemical oxidative polymerization method using ammonium per sulphate as an oxidant. Subsequently, this EB form of Tetraaniline was doped with $\mathrm{BF}_{3}$ under controlled experimental conditions. $\mathrm{BF}_{3}$ doped Tetraaniline shows a higher conductivity.

\section{Experimental}

N-phenyl-1, 4-phenylenediamine, $\mathrm{BF}_{3}$-Etherate (Aldrich) P-toluene sulphonic acid, Ammonium persulphate [APS, $\left(\mathrm{NH}_{4}\right)_{2} \mathrm{~S}_{2} \mathrm{O}_{8}$ ], Sodium hydroxide $(\mathrm{NaOH})$ were obtained from Merck Chemicals, India and used as received. N-phenyl-1, 4-phenylenediamine was purchased from Aldrich, India. Double distilled water was used throughout all the synthetic processes. All other reagents were analytical grade and used without further purification.

\section{Synthesis of tetramer of aniline}

Tetramer of aniline was synthesized via in situ chemical oxidative polymerization in acidic medium. In typical synthesis experiment, a 0.6 M N-phenyl-1, 4-phenylenediamine (NPPD) was dissolved in $1.0 \mathrm{M} \mathrm{HCl}$ solution and ultra-sonicated over $1 \mathrm{hr}$ and cooled to $0-5{ }^{\circ} \mathrm{C}$ in an ice bath. A pre-cooled equimolar solution of ammonium persulfate, $\left(\mathrm{NH}_{4}\right)_{2} \mathrm{~S}_{2} \mathrm{O}_{8}$, in $1.0 \mathrm{M} \mathrm{HCl}$ was slowly added drop wise into the acidified solution of NPPD with vigorous stirring at reaction temperature of $0-5{ }^{\circ} \mathrm{C}$ for $1 \mathrm{~h}$. The colour of the reaction mixer slowly turns from colourless to light blue and finally to dark green. The resulting of green colour solution is indicating the formation of tetramer of aniline in its emeraldine form. The reaction was allowed proceed for another 2-3 hrs at $0-5{ }^{\circ} \mathrm{C}$ and reaction mixer is allowed warm to room temperature. The dark green $\mathrm{HCl}$ doped tetra aniline was filtered and washed several times with distilled water to remove the unreacted oxidant and dedoped by stirring for $12 \mathrm{hrs}$ in $0.5 \mathrm{M} \mathrm{NAOH}$ solution. Finally, dedoped tetramer was filtered and dried under dynamic vacuum at $50{ }^{\circ} \mathrm{C}$.

\section{Preparation of $B F_{3}$ doped Tetraaniline}

Owing to high susceptibility of Lewis acid towards hydrolysis, doping was carried out strictly anhydrous conditions. $\mathrm{BF}_{3}$-Etherate, 1:1 complex of $\mathrm{BF}_{3}$ and diethyl ether was used for doping. 
In order to reduce the exposure to atmosphere, $\mathrm{BF}_{3}$-etherate was distilled in vacuum and distillate was collected directly over the emeraldine base tetraaniline powder. The reactivity of $\mathrm{BF}_{3}$ in $\mathrm{BF}_{3}$ -etherate complex is remarkably reduced, makes it a lot easier to handle. In order to achieve maximum doping, excess dopant was added and reaction mixture was left to equilibrate for 24 hours and after which un-reacted $\mathrm{BF}_{3}$-etherate was removed under dynamic vacuum at room temperature. Pumping for longer period of time, leads to partial dedoping.

\section{Characterization}

The UV-Visible absorption spectra of the samples were recorded on a Perkin-Elmer double beam LS-50 spectrophotometer. The samples were dissolved in dry dimethylsulphoxide (DMSO) and centrifuged to remove any undissolved polymer. The clear solutions were taken in quartz cuvettes. The infrared spectra were recorded over the range 4000 - $400 \mathrm{~cm}-1$ in a Perkin-Elmer Model SPECTRUM 1000 FTIR spectrometer. The powdered samples were mixed thoroughly with $\mathrm{KBr}$ and pressed into thin pellets. Powder X-ray diffraction (XRD) pattern were recorded on a Siemens D5005 x-ray diffractometer at 1 per min. Morphology of $\mathrm{BF}_{3}$ doped tetraaniline studied by scanning electron microscopy.

\section{Results and Discussion}

Figure 1 depicts the UV-Visible spectra of $\mathrm{BF}_{3}$ doped tetraaniline which is in complete agreement with literature reports. ${ }^{14-15}$ The UV-Visible absorption spectrum in dimethyl sulfoxide (DMSO) showed three distinct bands at 325, 450 and $633 \mathrm{~nm}$. Absorption peaks at 363 and $633 \mathrm{~nm}$ have been assigned to $\pi \rightarrow \pi^{*}$ transition of benzenoid rings in the tetraaniline chain and the transition caused by inter chain charge transfer from two adjacent benzenoid ring to the quinoid ring of tetraaniline chain respectively. With doping new feature at $450 \mathrm{~nm}$, confirmed the doping of $\mathrm{BF}_{3}$ tetraaniline. It is important to note that the same changes have also been observed in the case of proton doped tetraaniline.

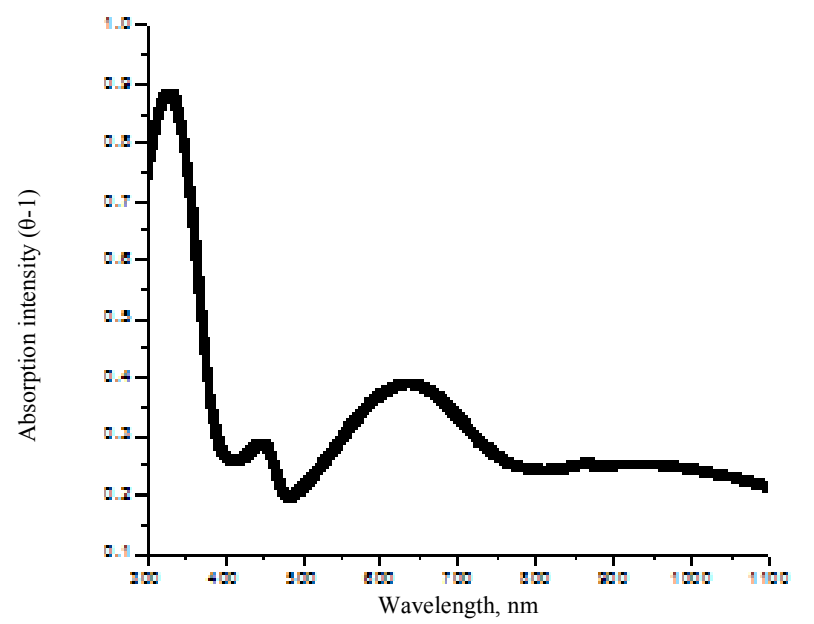

Figure 1. UV-Visible spectrum of $\mathrm{BF}_{3}$ doped tetraaniline.

Molecular structure of $\mathrm{BF}_{3}$ doped tetraaniline was characterized by X-Ray diffraction pattern (XRD) and Fourier-transform infrared (FTIR) spectroscopy. FTIR spectra of the nanocomposites are in agreement with the previous reports ${ }^{16-20}$. 
FTIR spectrum for $\mathrm{BF}_{3}$ doped tetraaniline is given in Figure 2. Peaks at 3184, 1610, and $1485 \mathrm{~cm}-1$ due to $\mathrm{N}-\mathrm{H}$ stretching vibration, $\mathrm{C}=\mathrm{C}$ stretching of quinoid phenyl and benzenoid phenyl rings, respectively. The peak at 1406 is due to stretching frequency of $\mathrm{B}-\mathrm{N}=\mathrm{Q}$ moiety (B refers to benzenoid and Q refers to quinoid ring).

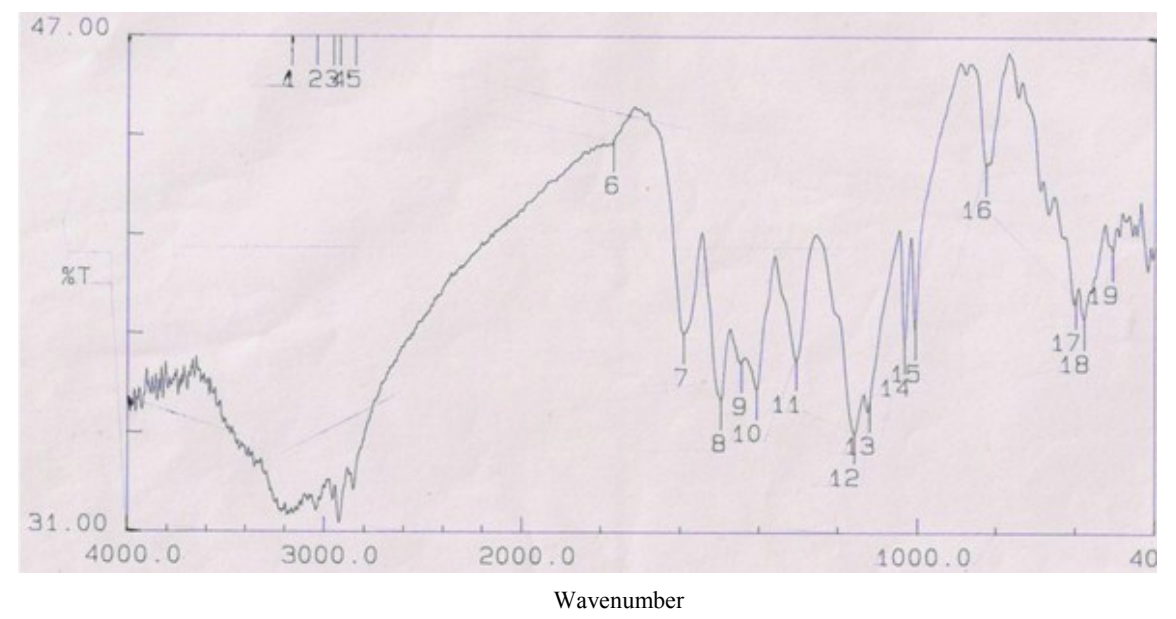

Figure 2. FTIR spectra of $\mathrm{BF}_{3}$ doped tetraaniline.

The presence of this peak confirms that the tetraaniline is doped with $\mathrm{BF}_{3}$. The peak at $1308 \mathrm{~cm}^{-1}$ is assigned to $\mathrm{C}-\mathrm{N}$ stretching vibrations of the 1 , 4- disubstituted benzene ring of tetraaniline. The peak at $833 \mathrm{~cm}^{-1}$ corresponds to $\mathrm{C}-\mathrm{H}$ out of plane bending of $1,4-$ disubstituted benzene rings of tetraaniline.

Figure 3, show the x-ray diffraction (XRD) patterns from undoped tetra aniline, $\mathrm{BF}_{3}$ doped tetraaniline and $\mathrm{HCl}$ doped tetraaniline. The XRD patterns of undoped tetraaniline is indicated that the amorphous in nature and completely agree with previous literature. The presence of a large number of sharp diffraction peaks upon doping with $\mathrm{HCl}$ indicated that the crystalline nature of $\mathrm{HCl}$ doped tetranailine.

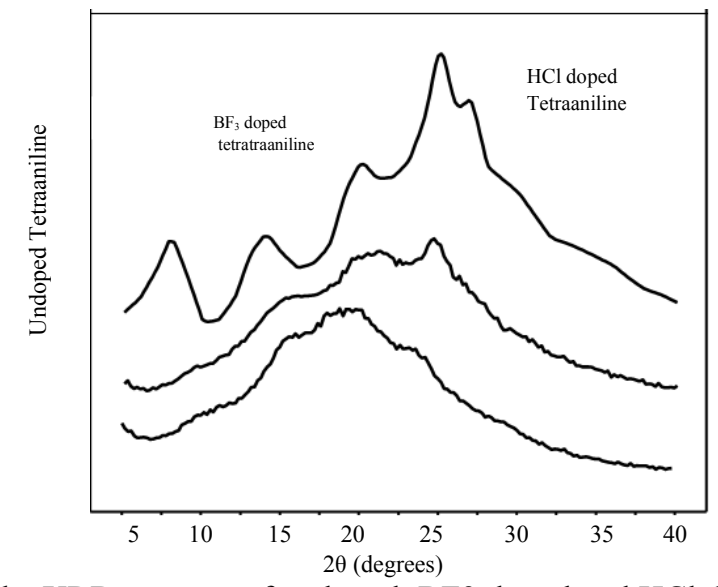

Figure 3. The XRD patterns of undoped, BF3 doped and $\mathrm{HCl}$ doped tetraaniline. 
In case of $\mathrm{BF}_{3}$ doped tetraaniline, diffractions peaks almost identical to undoped, amorphous tetraaniline. However, a closer inspection of the XRD pattern does indicated that the emergence of a weak intensity peak at $2 \theta \approx 24.8^{\circ}$. This peak position is exactly matches with the most intense peak in the $\mathrm{HCl}$ doped tetraaniline.
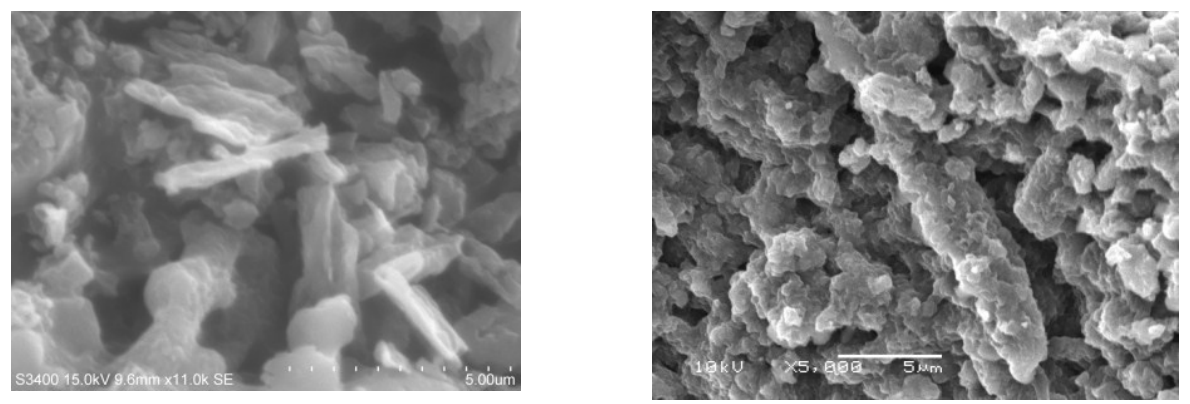

Figure 4. SEM image of Boron trifluoride $\left(\mathrm{BF}_{3}\right)$ doped Tetraaniline.

Morphology is invested by scanning electron microscopy. The SEM images of $\mathrm{BF}_{3}$ doped tetraaniline is shown in Figure 4. The SEM images confirm the nano-fibers morphology.

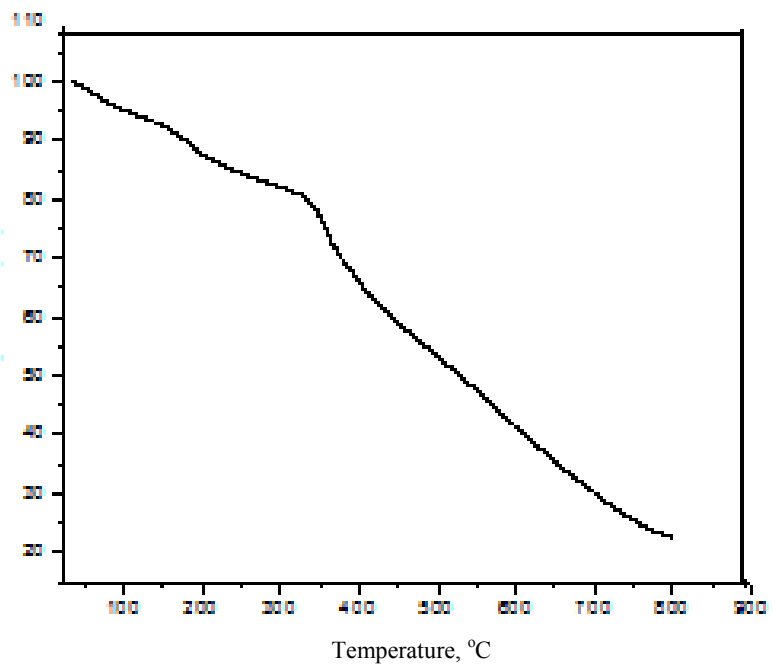

Figure 5. TGA curves of $\mathrm{BF}_{3}$ doped tetraaniline under $\mathrm{N}_{2}$ flow at a heating rate of $20{ }^{\circ} \mathrm{C}$ per minute.

Figure 5 shows a $\mathrm{TG}$ curve of $\mathrm{BF}_{3}$ doped tetraaniline prepared via oxidative polymerization of N-phenyl-1, 4-phenylenediamine under $\mathrm{N}_{2}$ flow at a heating rate of $20{ }^{\circ} \mathrm{C}$ per minute from $50-850{ }^{\circ} \mathrm{C}$.Three main inflection regions can be observed. The first region at lower temperatures $\left(<150{ }^{\circ} \mathrm{C}\right)$ is due to removal water, and other volatiles in $\mathrm{BF}_{3}$ doped tetra aniline. The weight loss in temperature range of $150-450{ }^{\circ} \mathrm{C}$ can be ascribed to the removal of $\mathrm{BF}_{3}$ and decomposition of tetraaniline chain. The third weight loss region of 450 - 
$840{ }^{\circ} \mathrm{C}$ is due to decomposition of tetraaniline chain. Thermal studies indicated that thermal decomposition of tetraniline chain occurs at higher temperature for $\mathrm{BF}_{3}$ doped tetraaniline as compared to pure tetraaniline.

\section{Conclusion}

In summary, we have demonstrated a simple, reproducible, and facile method of preparation of $\mathrm{BF}_{3}$ doped tetraaniline via oxidative chemical polymerization and followed by doping with $\mathrm{BF}_{3}$-etherate. The spectroscopic results indicated that $\mathrm{BF}_{3}$ is doped to tetraaniline. $\mathrm{TG}$ data indicated that $\mathrm{BF}_{3}$ doping enhanced the thermal stability of tetraaniline. The developed $\mathrm{BF}_{3}$ doped tetraaniline could have potential applications in organic transformation as polymer supported solid catalyst.

\section{References}

1. Unsworth J, Lunn B A, Innis P C, Jin Z, Kaynak A and Booth N G, J Intel Mat Syst Str., 1992, 3,380-95.

2. Schoch Jr K F, IEEE Electric Insulat Mag., 1994, 10, 29-32.

3. Angelopoulos M, IBM J Res Dev., 2001, 45, 57-75.

4. Gospodinova N and Terlemezyan L, Prog Polym Sci., 1998, 23, 1443-84.

5. $\quad \mathrm{Wu} \mathrm{C}$ G and Bein T, Science, 1994, 264, 1757.

6. Liang L, Liu J, Windisch C F, Exarhos G J and Lin Y, Angew Chem Int Ed., 2004,41, 3665 .

7. Liu H, Kameoka J, Czaplewski D A and Graighead H G, Nano Lett., 2004, 4, 671.

8. Virji S, Huang J, Kaner R B and Weiller B H, Nano Lett., 2004,4, 491.

9. Zhang D and Wang Y, Mater Sci Eng B, 2006, 134, 9.

10. Skotheim T A, Elsenbaumer R L and Reynolds J R, Handbook of Conducting Polymers, Marcel Dekker, New York, 1997.

11. Premamoy G, Samir K S and Amit C, Eur Polym J., 1999, 35, 699.

12. Genoud F, Kulszewicz-Bajer I, Bedel A, Jeandey C, Oddou J-L and Pron A, Chem Mater., 2000, 12, 744-749.

13. Kulszewicz-Bajer I, Pron A, Abramowicz J, Jeandey C, Oddou J-L and Sobczak J W, Chem Mater., 1999, 11, 552-556.

14. Venugopal G, Quan X, Johnson G E, Houlihan F M, Chin E and O.nalamasu, Ce Mate., 1995, 7, 271.

15. M. Hasik, I. Kurkowska, A. Bernasik, React Funct Polym., 2006, 66(12), 1703.

16. L. Zang, M. Wan, Y.Wei, Macromol Rapid Commun., 2006, 27, 366.

17. Chen S-A and Lee H T, Macromolecules, 1995, 28, 348.

18. Trhcova M, and Stejskal J and Prokes J, Synth Met., 1999,101, 840.

19. Neoh K G, Pun M Y, Kang E T and Tan K L, Synth Met., 1995,73,209.

20. Kim B-J, Oh S-G, Han M G and Im S-S, Langmuir, 2000, 16, 5841. 


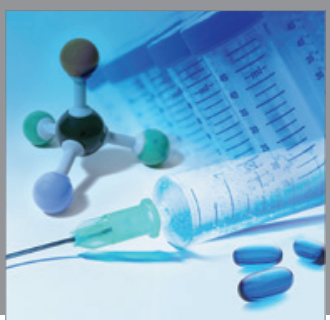

International Journal of

Medicinal Chemistry

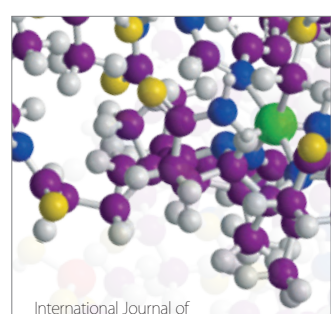

Carbohydrate Chemistry

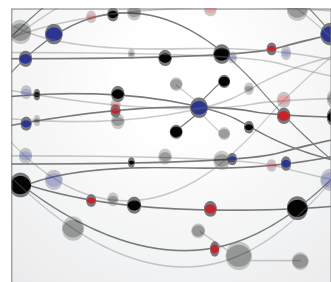

The Scientific World Journal
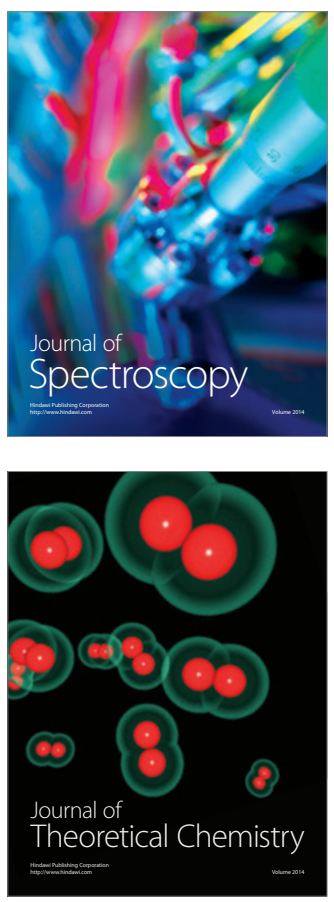
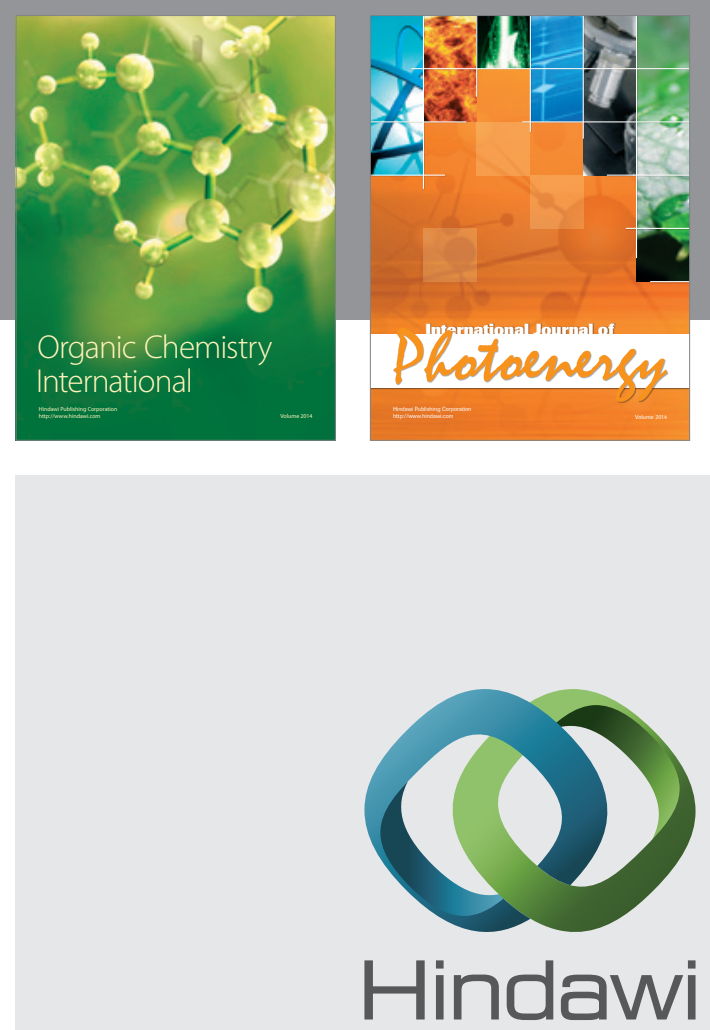

Submit your manuscripts at

http://www.hindawi.com
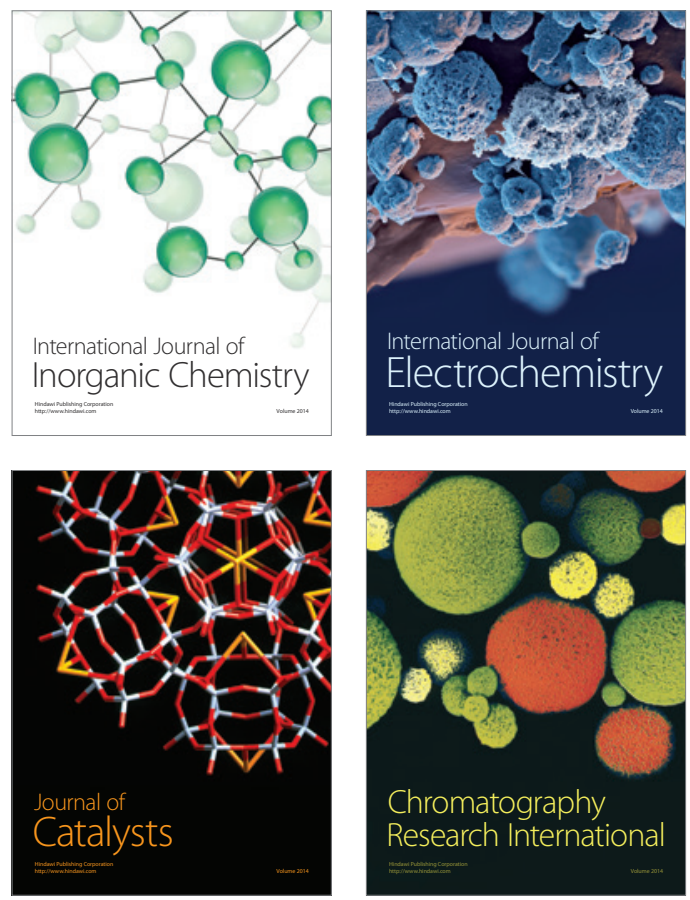
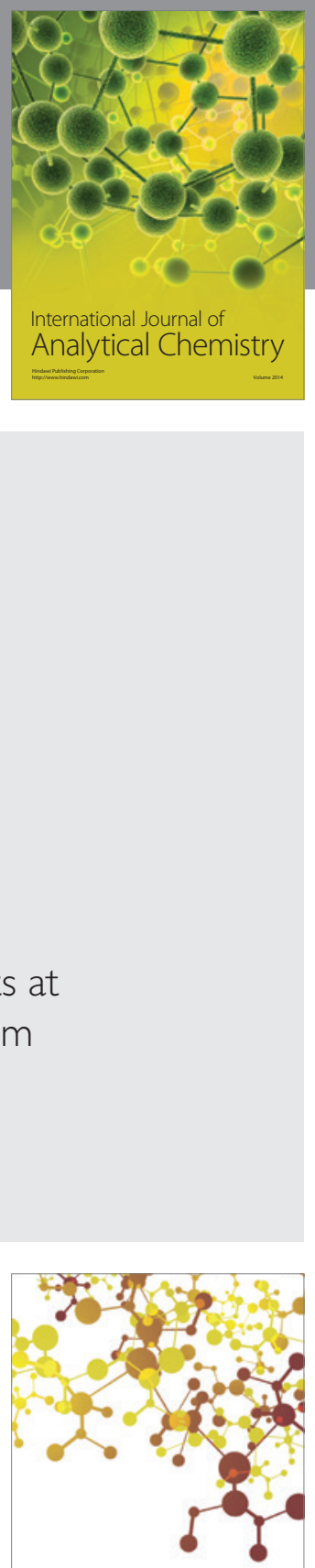

Journal of

Applied Chemistry
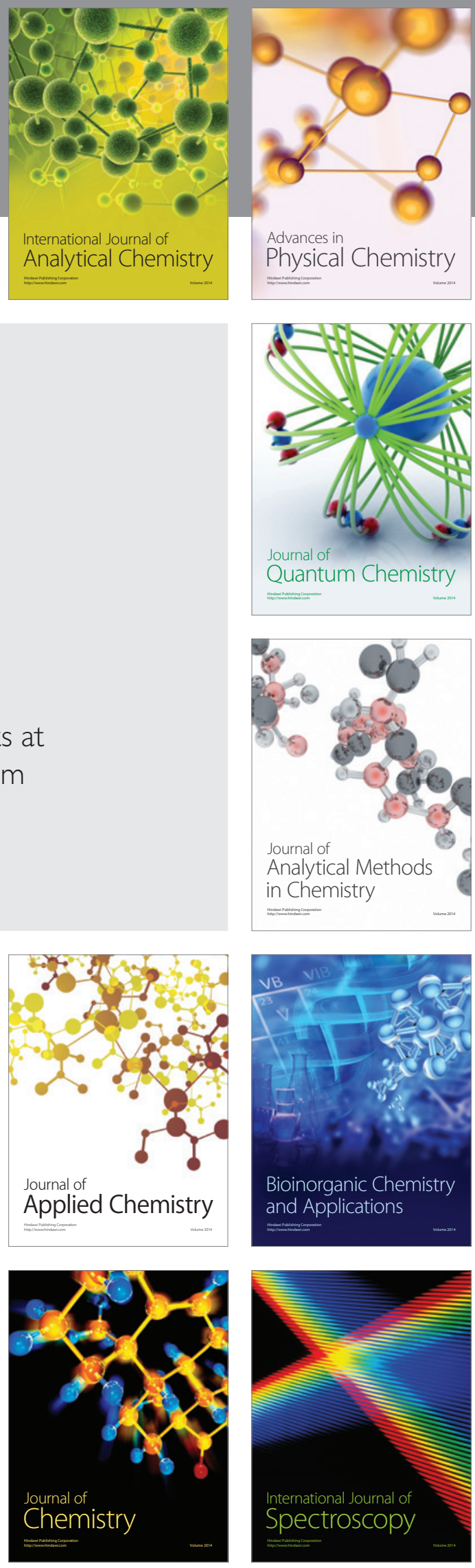